\title{
AN UNCONDITIONAL RESULT ABOUT GROTHENDIECK SPACES
}

\author{
RICHARD HAYDON
}

\begin{abstract}
It is shown that if $X$ is a nonreflexive Banach spach such that every weak* convergent sequence in $X^{*}$ is also weakly convergent, then $X^{*}$ has a subspace isometric to $L_{1}\left(\{0,1\}^{\omega_{1}}\right)$
\end{abstract}

It was shown in a recent article [4] that if the cardinal $\mathfrak{p}$ is greater than $\omega_{1}$ (which is the case if we assume Martin's Axiom and the negation of the Continuum Hypothesis), then every nonreflexive Grothendieck space contains a subspace isomorphic to $l_{1}(\mathfrak{p})$. It is known that this result is false if we assume the Continuum Hypothesis [6]. This paper presents a proof without special axioms that if $X$ is a nonreflexive Grothendieck space, then $X^{*}$ has a subspace isometric to $L_{1}\left(\{0,1\}^{\mathfrak{p}}\right)$. In fact, the hypotheses on $X$ in Theorem 1 are somewhat weaker, being those of the Hagler-Johnson theorem (Theorem 1(a) of [3]).

Notation and terminology for Banach spaces are standard. A Banach space $X$ is a Grothendieck space if every weak* convergent sequence in $X^{*}$ is also weakly convergent. For a set $I, L_{1}\left(\{0,1\}^{I}\right)$ denotes the $L_{1}$-space for the usual measure on $\{0,1\}^{I}$. We can regard this either as the infinite product of measures $\frac{1}{2}\left(\delta_{0}+\delta_{1}\right)$ on each coordinate, or as Haar measure on the compact group $\{0,1\}^{I}$.

Cardinals are as usual identified with initial ordinals; $\omega$ is the first infinite cardinal, and is also the set of natural numbers; $\omega_{1}$ is the first uncountable cardinal. The continuum $2^{\omega}$ is denoted by $c$ and the cardinal of a set $I$ by $\# I$.

The cardinal $\mathfrak{p}$ is the smallest cardinal such that there exists a family $\left(M_{\xi}\right)_{\xi \in \mathfrak{p}}$ of subsets of $\omega$ such that

(a) $\bigcap_{\xi \in Q} M_{\xi}$ is infinite for all finite $Q \subset \mathfrak{p}$;

(b) there is no infinite $M$ with $M \backslash M_{\xi}$ finite for all $\xi \in \mathfrak{p}$.

Rephrased succinctly, one can diagonalize fewer than $\mathfrak{p}$ compatible infinite subsets of $\omega$. An account of $\mathfrak{p}$ and its relation to other cardinals can be found in [1] or [2]. It is known, for instance, that $\mathfrak{p}$ is a regular cardinal with $\omega_{1} \leq \mathfrak{p} \leq \mathfrak{c}$.

We are now in a position to state our theorem.

THEOREM 1. Let $X$ be a Banach space and assume that in $X^{*}$ there is an infinite-dimensional subspace $Y$ such that every weak* convergent sequence in $Y$ is also norm convergent. Then $X^{*}$ has a subspace isometric to $L_{1}\left(\{0,1\}^{\mathfrak{p}}\right)$.

COROLlARY. If $X$ is a nonreflexive Grothendieck space, then $X^{*}$ has a subspace isometric to $L_{1}\left(\{0,1\}^{\mathfrak{p}}\right)$.

PROOF OF THE COROLlaRY. Since $X^{*}$ is not reflexive it contains a sequence with no weakly convergent subsequence. Since every weak Cauchy sequence is

Received by the editors May 12, 1986.

1980 Mathematics Subject Classification (1985 Revision). Primary 46B20; Secondary 46E30. 
weak* convergent, and hence weakly convergent, we see that our sequence has no weak Cauchy subsequence. So by Rosenthal's $l_{1}$ theorem [5], $X^{*}$ has a subspace isomorphic to $l_{1}$. By the Schur property of $l_{1}$ and the Grothendieck property of $X$, this subspace will do as $Y$ in the theorem.

The starting point for the proof of the theorem is the following lemma, taken from [3]. Recall that $g$ is said to be an $l_{1}$-normalized linear combination of $f_{n}$ $(n \in \omega)$ if there exist a finite subset $B \subset \omega$ and real numbers $\alpha_{n}(n \in B)$ such that

$$
g=\sum_{n \in B} \alpha_{n} f_{n} \text { and } 1=\sum_{n \in B}\left|\alpha_{n}\right|
$$

We say that $\left(g_{n}\right)$ is an $l_{1}$-normalized block-subsequence of $\left(f_{n}\right)$ if each $g_{n}$ is an $l_{1}$-normalized linear combination

$$
g_{n}=\sum_{r \in B_{n}} \alpha_{r} f_{r}
$$

and $\max B_{n}<\min B_{n+1}$.

LEMMA 1 (HAGLER-JOHNSON). Let $X$ be a Banach space and suppose that in $X^{*}$ there is an infinite-dimensional subspace $Y$ such that every weak* convergent sequence in $Y$ is also norm convergent. Then there exists a bounded sequence $\left(f_{n}\right)$ in $X^{*}$ such that

$$
\sup _{x \in \text { ball } X} \limsup _{n \rightarrow \infty}\left\langle g_{n}, x\right\rangle=1
$$

for every $l_{1}$-normalized block subsequence $\left(g_{n}\right)$ of $\left(f_{n}\right)$.

The idea for Lemma 2 can also be found in [3].

LEMMA 2. Suppose that $\left(f_{n}\right)$ is as in Lemma 1. Let $p \geq 1$ be a natural number and let $g_{i j}(1 \leq i \leq p, j \in \omega)$ be $l_{1}$-normalized linear combinations of the $f_{n}$,

$$
g_{i j}=\sum_{r \in B_{i j}} \alpha_{r} f_{r}
$$

such that

$$
B_{i j} \cap B_{k l}=\varnothing \quad \text { unless }(i, j)=(k, l) .
$$

Then, for all $\eta<1$, there exists an infinite subset $M$ of $\omega$, together with elements $x^{F}(F \subseteq\{1,2, \ldots, p\})$ of ball $X$, such that, for all $j \in M$.

$$
\left\langle g_{i, j}, x^{F}\right\rangle \begin{cases}>\eta & \text { if } i \in F, \\ <-\eta & \text { if } i \notin F .\end{cases}
$$

PrOOF. For each $E \subseteq\{1,2, \ldots, p\}$, we consider

$$
h_{j}^{E}=\frac{1}{p}\left[\sum_{i \in E} g_{i, j}-\sum_{i \notin E} g_{i, j}\right] .
$$

By our assumptions about the $g_{i, j}$ some subsequence of $\left(h_{j}^{E}\right)$ is an $l_{1}$-normalized block subsequence of $\left(f_{n}\right)$. Thus, given $E$ and $\varepsilon>0$, we may find an infinite subset $K$ of $\omega$ and $x^{E} \in$ ball $X$ such that

$$
\left\langle h_{j}^{E}, x^{E}\right\rangle>1-\varepsilon
$$


for all $j \in K$. By repeating this process $2^{p}$ times, we obtain an infinite subset $L$ of $\omega$ and elements $x^{F} \in$ ball $X$ such that

$$
\left\langle h_{j}^{F}, x^{F}\right\rangle>1-\varepsilon
$$

for all $F$ and all $j \in L$.

Now for any $F$, the set of $j$ such that there exists $i \leq p$ with

$$
\left|\left\langle g_{i, j}, x^{F}\right\rangle\right|>1+\varepsilon
$$

is finite. Thus, we may delete a finite number of elements of $L$ to obtain a set $M$ such that

$$
\left\langle h_{j}^{F}, x^{F}\right\rangle>1-\varepsilon \text { and }\left|\left\langle g_{i j}, x^{F}\right\rangle\right| \leq 1+\varepsilon
$$

for all $j \in M$ and all $F \subseteq\{1,2, \ldots, p\}$. It is easy to see that these inequalities imply

$$
\left\langle g_{i, j}, x^{F}\right\rangle>1-(2 p-1) \varepsilon
$$

when $i \in F$, and

$$
\left\langle g_{i, j}, x^{F}\right\rangle<-1+(2 p-1) \varepsilon
$$

when $i \notin F$. So, given an appropriate initial choice of $\varepsilon$, we have the result.

As a first step towards proving the theorem, let us see what we need to do in order to define an isometry from $L_{1}\left(\{0,1\}^{I}\right)$ into a Banach space $Y$. We write $P_{f}(I)$ for the set of all finite subsets of $I, \mathcal{F}_{I}$ for the union $\left.\bigcup\{0,1\}^{A}: A \in \mathcal{P}_{f}(I)\right\}$ and, for $\alpha \in \mathcal{F}_{I}$, we let $e_{\alpha}$ be the element of $L_{1}\left(\{0,1\}^{I}\right)$ defined by

$$
e_{\alpha}(z)= \begin{cases}2^{\# \operatorname{dom} \alpha} & \text { if } z \mid \operatorname{dom} \alpha=\alpha \\ 0 & \text { if not. }\end{cases}
$$

The linear span of the $e_{\alpha}\left(\alpha \in \mathcal{F}_{I}\right)$ is dense in $L_{1}\left(\{0,1\}^{I}\right)$ and we have

$$
e_{\alpha}=s^{\# A-\# B} \sum_{\substack{\beta \in\{0,1\}^{B} \\ \beta \mid A=\alpha}} e_{\beta}
$$

when $A=\operatorname{dom} \alpha \subset B$. The following lemma is easy to prove.

LEMMA 3. Let $Y$ be a Banach space, $I$ be a set, and let $\left(h_{\alpha}\right)_{\alpha \in \mathcal{F}_{I}}$ be a family of elements of $Y$. Then there exists a bounded linear operator $T: L_{1}\left(\{0,1\}^{I}\right) \rightarrow Y$ with $T e_{\alpha}=h_{\alpha}\left(\alpha \in \mathcal{F}_{I}\right)$ if and only if the family $\left(h_{\alpha}\right)$ is bounded and the following condition is satisfied:

$$
h_{\alpha}=2^{\# A-\# B} \sum_{\substack{\beta \in\{0,1\}^{B} \\ \beta \mid A=\alpha}} h_{\beta}
$$

whenever $\operatorname{dom} \alpha=A \subset B \in \mathcal{P}_{f}(I)$.

The operator $T$ is isometric if and only if we have

$$
\left\|\sum_{\alpha \in\{0,1\}^{A}} \theta(\alpha) h_{\alpha}\right\|=\sum_{\alpha \in\{0,1\}^{A}}|\theta(\alpha)|
$$

whenever $A \in \mathcal{P}_{f}(I)$ and $\theta:\{0,1\}^{A} \rightarrow \mathbf{R}$.

In the proof of the theorem, we shall obtain vectors $h(\alpha)$ as weak* limits of certain $h_{n}(\alpha)$. The key combinatorial idea is that of a dyadic system. 
Let $D_{j}(j \in \omega)$ be the sequence of sets $D_{0}=\{1\}, D_{1}=\{2,3\}, \ldots, D_{j}=$ $\left\{2^{j}, 2^{j}+1, \ldots, 2^{j+1}-1\right\}, \ldots$ and let $I$ be a set. Suppose that, for each $a \in I, P_{j}(a)$ is a subset of $D_{j}$. For each finite subset $A$ of $I$, let $M(A)$ be the set of all $j \in \omega$ such that, for all $\alpha \in\{0,1\}^{A}$, the intersection

$$
\bigcap_{\substack{a \in A \\ \alpha(a)=0}} P_{j}(a) \cap \bigcap_{\substack{a \in A \\ \alpha(a)=1}}\left(D_{j} \backslash P_{j}(a)\right)
$$

has exactly $2^{j-\# A}$ elements. We say that $\left(P_{j}(a)\right)$ forms a dyadic system over $I$ if $\omega \backslash M(A)$ is finite for each finite $A \subseteq I$.

Let us note that there exists a dyadic system over a set $I$ with $\# I=\mathfrak{c}$.

LEMMA 4. There exists a dyadic system $\left(P_{j}(z)\right)$ indexed by $z \in\{0,1\}^{\omega}$.

PROOF. First of all, fix, for each $j>0$, a family $Q_{j, r}(0 \leq r<j)$ of subsets of $D_{j}$ such that

$$
\# \bigcap_{r \in H} Q_{j, r}=2^{j-\# H}
$$

for every $H \subseteq\{0,1, \ldots, j-1\}$. The obvious choice is

$$
Q_{j, r}=\left\{2^{j}+m: 0 \leq m<2^{j} \&\left[2^{-r} m\right] \text { is even }\right\} .
$$

We shall put

$$
P_{j}(z)=Q_{j, r(z, j)}
$$

for suitably chosen $r(z, j)$. What we need is that, for distinct $w, z \in\{0,1\}^{\omega}$, $r(z, j)=r(w, j)$ for only finitely many values of $j$. This can be achieved if, for example, we put $l=\left[\log _{2} j\right]$ and

$$
r(z, j)=\sum_{m=0}^{l-1} z_{m} 2^{m}
$$

PROOF OF THE THEOREM. Let $\left(P_{j}(\xi)\right)_{\xi \in \mathfrak{p}}$ be a dyadic system over $\mathfrak{p}$, where we regard $\mathfrak{p}$ as the set of ordinals $\xi<\mathfrak{p}$. Such a system exists since $\mathfrak{p} \leq \mathfrak{c}$. For $A \in \mathcal{P}_{f}(\mathfrak{p}), \alpha \in\{0,1\}^{A}$ write

$$
D_{j}(\alpha)=\bigcap_{\substack{a \in A \\ \alpha(a)=0}} P_{j}(a) \cap \bigcap_{\substack{a \in A \\ \alpha(a)=1}}\left(D_{j} \backslash P_{j}(a)\right)
$$

and

$$
h_{j}(\alpha)=\frac{1}{\# D_{j}(\alpha)} \sum_{n \in D_{j}(\alpha)} f_{n}
$$

We shall use transfinite induction to define infinite subsets $M_{\xi}$ of $\omega(\xi \in \mathfrak{p})$ and elements $x(F)$ of ball $X\left(F \subseteq\{0,1\}^{A}, A \in \mathcal{P}_{f}(\mathfrak{p})\right)$. These will have the properties:

(i) $M_{\eta} \backslash M_{\xi}$ is finite when $\xi<\eta \in \mathfrak{p}$.

(ii) For all $\xi \in \mathfrak{p}$, all $A \in \mathcal{P}_{f}(\xi)$, and all $F \subseteq\{0,1\}^{A}$, there is a finite $L \subset M_{\xi}$ such that, for $j \in M_{\xi} \backslash L$,

$$
\left\langle h_{j}(\alpha), x(F)\right\rangle \begin{cases}>1-1 / \# A & \text { if } \alpha \in F \\ <-1+1 / \# A & \text { if } \alpha \in\{0,1\}^{A} \backslash F .\end{cases}
$$


To do the recursive construction, suppose that $M_{\xi}$ and $x(F)$ have been defined already for all $\xi<\eta$ and all $F \subseteq\{0,1\}^{A}\left(A \in \mathcal{P}_{f}(\xi)\right)$. If $\eta$ is a limit ordinal, we use the fact that $\eta<\mathfrak{p}$ to find an infinite $M_{\eta} \subset \omega$ with $M_{\eta} \backslash M_{\xi}$ finite for all $\xi<\eta$. Evidently, no new $x(F)$ need to be defined in this case.

Next, let $\eta$ be a successor ordinal, $\eta=\varsigma+1$. We have to find elements $x(F)$ $\left(F \subseteq\{0,1\}^{A \cup\{\varsigma\}}, A \in \mathcal{P}_{f}(\varsigma)\right)$, and obtain the subsequence $M_{\zeta+1}$. We note that, given $A \in P_{f}(\zeta)$ and an infinite subset $M$ of $\omega$, we can apply Lemma 2 (with $\left.p=2^{\#(A \cup\{s\})}\right)$ to obtain an infinite subset $M^{\prime}$ of $M$ and vectors $x(F) \in$ ball $X$ $\left(F \subseteq\{0,1\}^{A \cup\{s\}}\right)$ such that, for $j \in M^{\prime}$,

$$
\left\langle h_{j}(\alpha), x(F)\right\rangle \begin{cases}>1-1 / \# A & \text { if } \alpha \in F, \\ <-1+1 / \# A & \text { if not. }\end{cases}
$$

We now well-order $P_{f}(\zeta)$ and repeatedly use the above observation, together with the fact that $\# P_{f}(\zeta)<\mathfrak{p}$. We obtain in this way an infinite subset $M_{\varsigma+1}$ of $M_{\zeta}$, together with elements $x(F)$ of ball $X$ and finite subsets $L(F)$ of $M_{\varsigma+1}$ (for $F \subseteq$ $\left.\{0,1\}^{A \cup\{\varsigma\}}, A \in P_{f}(\zeta)\right)$, in such a way that (*) holds whenever $j \in M_{\zeta+1} \backslash L(F)$.

Once the sets $M_{\xi}$ and the vectors $x(F)$ have been obtained, we let $\mathcal{U}$ be an ultrafilter on $\omega$ which contains all the sets $M_{\xi}$ and all the cofinite sets. We define $h(\alpha)=\mathrm{w}^{*} \lim _{j \rightarrow u} h_{j}(\alpha)$ and only have to check that (a) and (b) of Lemma 3 are satisfied. have

If $A \subset B \in \mathcal{P}_{f}(\mathfrak{p})$ and $\alpha \in\{0,1\}^{A}$ then for all $j$ in the cofinite set $M(B)$, we

$$
h_{j}(\alpha)=2^{\# A-\# B} \sum_{\substack{\beta \in\{0,1\}^{B} \\ \beta \mid A=\alpha}} h_{j}(\beta)
$$

because of the definition of dyadic system.

Taking the limit as $j \rightarrow \mathcal{U}$ gives (2).

Now if $A \in \mathcal{P}_{f}(\mathfrak{p})$ and $\theta:\{0,1\}^{A} \rightarrow \mathbf{R}$ we may fix any $B \in \mathcal{P}_{f}(\mathfrak{p})$ with $B \supseteq A$ and consider

$$
G=\left\{\beta \in\{0,1\}^{B}: \theta(\beta \mid A)>0\right\} .
$$

It is easy to check that, if $B \subseteq \xi \in \mathfrak{p}$,

$$
\left\langle\sum_{\alpha \in\{0,1\}^{A}} \theta(\alpha) h_{j}(\alpha), x(G)\right\rangle \geq(1-1 / \# B) \sum_{\alpha}|\theta(\alpha)|
$$

for all but finitely many $j \in M_{\xi}$.

This tells us that

$$
\left\|\sum \theta(\alpha) h(\alpha)\right\| \geq(1-1 / \# B) \sum|\theta(\alpha)| .
$$

Since \# $B$ may be chosen to be an arbitrarily large integer, we have proved (b).

REMARKS. Although the proof has been presented here for the cardinal $\mathfrak{p}$, the only point of interest of the result lies in the case $\mathfrak{p}=\omega_{1}$. If $\mathfrak{p}>\omega_{1}$, then the result of [4] is in two respects stronger than the one given here. First, the hypothesis in [4] is that there exists in $X^{*}$ a bounded sequence with no weak* convergent convex block subsequence; here we need " $l_{1}$-normalized" instead of "convex." Secondly, the conclusion in [4], that $X$ contains $l_{1}(\mathfrak{p})$, implies that $X^{*}$ has a subspace isometric to $C\left(\{0,1\}^{\mathfrak{p}}\right)^{*}$. I do not know whether it is true unconditionally that the dual of a 
nonreflexive Grothendieck space contains $C\left(\{0,1\}^{\omega_{1}}\right)^{*}$. I also do not know whether the convex block hypothesis of [4] suffices for an unconditional result.

\section{REFERENCES}

1. E. K. van Douwen, The integers and topology, Handbook of Set-Theoretic Topology (edited by K. Kunen and J. Vaughan), Elsevier, New York, 1984.

2. D. H. Fremlin, Consequences of Martin's axiom, Cambridge Univ. Press, 1985.

3. J. Hagler and W. B. Johnson, On Banach spaces whose dual balls are not weak* sequentially compact, Israel J. Math. 28 (1977), 325-330.

4. R. Haydon, M. Levy and E. Odell, On sequences without weak* convergent convex block subsequences, Proc. Amer. Math. Soc. 100 (1987), 94-98.

5. H. P. Rosenthal, A characterization of Banach spaces containing $l_{1}$, Proc. Nat. Acad. Sci. U.S.A. 71 (1974), 2411-2413.

6. M. Talagrand, Un nouveau $C(K)$ qui possède la propriété de Grothedieck, Israel J. Math. 37 (1980), 181-191.

Brasenose College, Oxford, England 